\title{
Rational development of telehealth to support primary care respiratory medicine: patient distribution and organisational factors
}

\author{
David John Goddena, *Gerry King ${ }^{a}$ \\ ${ }^{a}$ Centre for Rural Health, Centre for Health Science, University of Aberdeen, Inverness, UK
}

Originally submitted 23rd July 2010; resubmitted 23rd November 2010; revised 22nd April 2011; accepted 2nd May 2011; online 22nd July 2011

\begin{abstract}
Aims: Telehealth may offer opportunities to support primary and secondary care of patients with respiratory disease. This study examined the potential for applying telehealth in a region of the UK by exploring the distribution of patients and examining attitudes to implementation of telehealth.

Methods: The distribution of patients with asthma, COPD, lung cancer and obstructive sleep apnoea (OSAS) in the NHS Highland Region (309,900 residents, 12,507 square miles) was determined from Quality and Outcomes Framework data and disease registers. Qualitative interviews with health professionals $(n=20)$ focussing on the potential for telehealth in respiratory medicine were analysed using the Normalisation Process Model.

Results: The most remote general practices accounted for $40 \%$ of patients with asthma (7198/17822), 45\% of those with COPD (2145/4721), 33\% of lung cancer (199/605) and 35\% of OSAS (169/489) patients. Urban figures were $28 \%$ of asthma patients, $26 \%$ of COPD patients, $25 \%$ of lung cancer and $31 \%$ of OSAS patients. Interviewees identified a range of telehealth applications they considered potentially beneficial including management, information and communication systems. However, they also identified challenges - mainly related to training, costs and infrastructure.

Conclusions: Tailoring telehealth to support management of respiratory diseases in primary care requires knowledge of patient distribution, which will impact on the nature and feasibility of services. Individual and organisational capacities and attitudes are also likely to influence successful implementation.

(C) 2011 Primary Care Respiratory Society UK. All rights reserved.

DJ Godden and G King. Prim Care Respir J 2011; 20(4): 415-420

http://dx.doi.org/10.4104/pcrj.2011.00063
\end{abstract}

Keywords telehealth, asthma, COPD, sleep apnoea, lung cancer, primary care, patient distribution

See linked editorial by Morrison and Mair on pg 351

\section{Introduction}

Respiratory conditions such as asthma, chronic obstructive pulmonary disease (COPD), lung cancer and obstructive sleep apnoea syndrome (OSAS) are important causes of morbidity and mortality. ${ }^{1}$ Telehealthcare offers opportunities to bridge primary and secondary care by allowing care to be delivered remotely with electronic transmission of information. ${ }^{2}$ It encompasses electronic patient records, tele-consultation between patients and practitioners and between health professionals, real time transmission of information from patients in the community, and bespoke systems for remote management of respiratory disease including monitoring of specific physiologic parameters.

Mair et al. describe four domains of telehealth: management systems; information systems; communication systems; and decision support. ${ }^{3}$ Telehealth may have particular potential in dispersed communities, aligning with health policy to provide care as locally as possible with access to specialist care as necessary, ${ }^{4}$ recognising that distance from services is a barrier to uptake. ${ }^{5}$ However, while recent articles have discussed the potential for telehealth in respiratory disease, they have also pointed out a lack of published robust evidence of clinical and economic benefit. 2,6,7

\footnotetext{
* Corresponding author: Ms Gerry King, University of Aberdeen, Centre for Rural Health, Centre for Health Science, Old Perth Road, Inverness, IV2 3JH, UK. Tel: +44 (0)1463 255902 Fax: +44 (0)1463 255802 E-mail: gerry.king@abdn.ac.uk
} 
The feasibility of telecare and telemonitoring for COPD and asthma has been demonstrated, ${ }^{8-12}$ and telemonitoring has been reported to reduce hospital admissions, emergency department visits and length of stay. ${ }^{13,14}$ Remote monitoring of chemotherapy by mobile phone has also proven successful in lung cancer management. ${ }^{15}$ In a systematic review of telehealth applications, patient acceptance and satisfaction were generally high, although robust evidence on cost effectiveness and benefits was lacking. ${ }^{16}$ For asthma, a more recent systematic review of randomised controlled trials of telehealthcare highlighted the importance of disease severity in determining effectiveness - clinical benefit being more likely in those at greater risk of hospital admission. ${ }^{17}$ Telehealth might prove attractive in rural areas where travel times and costs are high. Telemedicine was cost-effective for delivery of outpatient pulmonary care for rural populations with limited access to subspecialty services in the USA, ${ }^{18}$ while in Finland, an e-mail triage system supported by teleconsultation reduced costs and demand for hospital outpatient care. ${ }^{19}$

However, despite the potential offered by telehealth, widespread uptake has not occurred in the UK. Problems with implementation often relate to failure to integrate telehealth systems into standard clinical practice, ${ }^{20}$ while in rural settings, lack of connectivity to broadband and mobile networks may be a further barrier.

Establishing the distribution of potential patients and the willingness of care providers to adopt telehealth are important prerequisites to implementation. We explored these issues in our region. To address potential demand, we examined the distribution of patients with common respiratory diseases across primary care. We then interviewed a range of health professionals across primary and secondary care to explore potential demand for telehealth and to identify factors that might influence successful implementation.

\section{Methods}

\section{Setting}

The study was conducted in NHS Highland, Scotland, which serves 309,900 residents across 12,507 square miles, augmented by many tourists. The main hub is the City of Inverness, while the area has mountainous terrain, a rugged coastline, and a number of populated islands. Primary care is delivered from 102 general practices, and specialist services from a 500-bed district hospital in Inverness and three small rural general hospitals.

\section{Patient distribution}

The number of patients with asthma and COPD at individual practice level was mapped from Quality Outcomes Framework [QOF] data ${ }^{21}$ for the 2006-7 financial year. These anonymised data indicate patients considered by their general practitioner (GP) to have the relevant diagnosis but they do not specify severity. Lung cancer data were collected from the cancer audit database at the district hospital for the period 1st January 2003 - 31st December 2007, although data from one part of the region, Argyll \& Bute, were available only from 2004 - 2007. Services for OSAS are provided from the district hospital, and all current patients in the period April-May 2008 were identified. Disease frequencies were examined in relation to the Clinical Peripherality [CP] Index, ${ }^{22}$ which reflects remoteness of rural practices from central services and the size and density of the population served. Indices were expressed in quintiles, CP 5 containing the most peripheral practices; urban practices were designated CP 0.

\section{Qualitative interviews}

We wished to determine, from a range of health professionals, aspects of respiratory care where they considered telehealth may have a role. Semi-structured interviews were conducted with health professionals and managers likely to be involved in implementation of telehealth. These key informants - including GPs, consultants, nurses, and others involved in respiratory care - had responsibilities across the region (including urban, rural and remote areas) and they were recruited purposively. The interview schedule asked about the scope of telehealth, previous experience with telehealth, and views on future potential initiatives. Each interview was recorded, transcribed and analysed using framework methodology. ${ }^{23}$ Telehealth applications were classified according to Mair et al. ${ }^{3}$ Interview data were transcribed verbatim.

Analysis was carried out using a 4-stage process. Using the initial themes, the interview schedule and the wider literature, a coding schedule was constructed. The codes were entered into the qualitative data analysis programme ' $N V i v o$ ' ${ }^{\oplus}$, which was used to organise the data and structure the analysis. Once the codes were applied the data were grouped into categories, and thematic analysis was performed looking for similar and divergent perspectives. The principles of the Normalisation Process Model described by May et al. ${ }^{24}$ were then used to explore how successful implementation of proposed new technologies could be achieved. This model assesses the conditions necessary to support the introduction of complex interventions into the health care workplace. ${ }^{24,25}$ It is defined by four constructs:

- interactional workability: how a complex intervention might affect interactions between people and practices

- relational integration: how the intervention relates to existing knowledge and relationships

- skill-set workability: how the intervention affects the current division of labour

- contextual integration: how the intervention relates to the organisation in which it is set.

The North of Scotland Research Ethics Service reviewed the study and advised that they considered it as service planning. 
Table 1. Distribution of patients by clinical peripherality

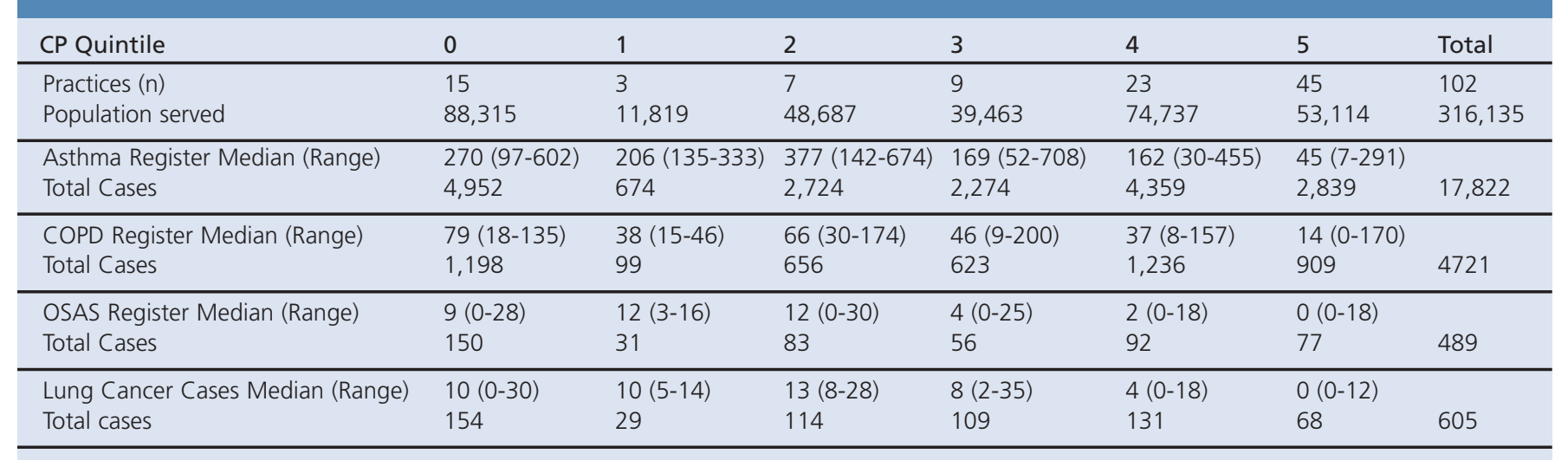

Distribution of all patients and patients with asthma, COPD, OSAS and lung cancer in practices is arranged according to Clinical Peripherality (criteria: remoteness, size, density; $C P O=$ urban, $C P 5=$ most peripheral

\section{Results}

Table 1 summarises the practice population data. As expected, the most peripheral practices had the smallest numbers of patients per practice with each condition. However, when total cases at each level of peripherality were examined, the most peripheral practices [CP quintiles 4 and 5] accounted for $40 \%$ (7198) of patients with asthma, 45\% (2145) of those with COPD, and 35\% (169) of OSAS and 33\% (199) of lung cancer patients. This compares with urban values of $28 \%$ (4952) of asthma patients, 26\% (1198) of COPD patients, and 25\% (154) of lung cancer and $31 \%$ (150) of OSAS patients.

All of those invited to interviews to examine the potential for telehealth $(n=20)$ agreed to take part. Thirteen were women, five worked exclusively in remote and rural areas, while the others provided services across the region for urban, rural and remote patients. Interviewees included two rural GPS, three community and practice nurses, four hospital consultants, four hospital nurses, five managers, one telehealth equipment supplier, and one allied health professional.

Respondents identified potentially beneficial applications in three of the four domains of telehealth. Communication systems were dominant. Decision support did not feature [see Table 2].

\section{Normalisation issues for telehealth applications}

Given the wide range of potential applications identified, the normalisation process issues varied between applications. The general normalisation themes for the main technologies discussed are summarised in Table 3.

Interactional workability is concerned with 'congruence' of the intervention and 'disposal' or effects of the interaction created. This was considered to be high for all of the technologies listed above. For example, in relation to interprofessional communication:

".....if I knew that at 9.30am every Wednesday I could call into one of the chest guys and speak to them, then I think it might work two ways; it may prevent some unnecessary referrals but it may also expedite some important referrals too ...." RT13

Another respondent highlighted the potential to reduce patient travel to hospitals:

"They'd still get that secondary care but they wouldn't have to have these horrendous journeys to get there and then be admitted overnight for no reason at all

Table 2. Telehealth applications by domain as addressed by interviewees

Management Systems

- Clinical management system for chest medicine

- Electronic patient record

Communication Systems

- Email

- Videoconferencing which include:

- Community Hospital to Consultant

- GP to Consultant

- Nurse to Consultant

- Nurse to Patient

- Remote health monitoring (with or without web cam)

- Videoconferencing for education

- Video link to ward for patients

- Pulmonary rehab into patients homes

- Multidisciplinary team/Managed Clinical Network for COPD

- Videoconferencing for supervision of physiotherapy rehabilitation

- Text reminders for appointments

- Video link for spirometry/respiratory function test training/supervision

Electronic transfer of data from Variable Positive Airway Pressure \& Continuous Positive Airway Pressure machines

\section{Information Systems}

- COPD E learning resource

- Respiratory website

- DVDs for education (GPS)

Decision Support

Did not feature 


\begin{tabular}{|c|c|c|c|c|}
\hline Technology & $\begin{array}{l}\text { Interactional } \\
\text { Workability }\end{array}$ & $\begin{array}{l}\text { Skill-set } \\
\text { Workability }\end{array}$ & $\begin{array}{l}\text { Contextual } \\
\text { Integration }\end{array}$ & $\begin{array}{l}\text { Relational } \\
\text { Integration }\end{array}$ \\
\hline SMS text reminders for appointments & +++ & $+/-$ & $\mathrm{N}-\mathrm{D}$ & +++ \\
\hline Email, mobile and phone technology & +++ & $+/-$ & $\mathrm{N}-\mathrm{D}$ & +++ \\
\hline Videoconferencing Hospital/Hospital & +++ & $+/-$ & $+/-$ & $+/-$ \\
\hline Learning at a Distance & +++ & $\mathrm{N}-\mathrm{D}$ & $\mathrm{N}-\mathrm{D}$ & +++ \\
\hline Remote health monitoring/Telemonitoring & +++ & $+/-$ & $+/-$ & $+/-$ \\
\hline Electronic transfer of data from CPAP and VPAP & +++ & $+/-$ & $+/-$ & $+/-$ \\
\hline Videoconferencing GP to consultant & +++ & $+/-$ & $+/-$ & $+/-$ \\
\hline Videoconferencing for Pulmonary rehabilitation & +++ & $\mathrm{N}-\mathrm{D}$ & $+/-$ & +++ \\
\hline
\end{tabular}

other than that they had to travel." RT08

The potential for telemonitoring to identify early exacerbations of disease was also noted:

"...I think some patients are very sensitive to changes in their physiological state but others are not at all, there's been a lot of work on asthma on poor perceivers, some people in asthma ...feel different when their peak flow drops, they're easy, they'll feel bad, they'll seek help. You have other people whose peak flow can drop by 50\% and they don't notice...we'll only end up seeing them when they're nearly dead ...". (RT07)

Relational integration, which is concerned with accountability and confidence, was generally positive for most interventions discussed. However, for videoconferencing and remote monitoring there were uncertainties about effectiveness and the quality of interactions compared to face-to-face meetings or consultations that affected confidence, particularly with life threatening illness:

"...we've got a lot of lung cancer sufferers as I'm sure you know...and it's just lots of non-verbal communication that's just very helpful, some of it yes, you would pick up if there was a big TV screen there, but even the wife sitting behind the husband who shakes her head at some of the answers he gives, or if I'm being softly, softly with something and the husband isn't getting it and you can see the look of absolute horror come across the wife's face because she's realised what we're gradually building up to, whereas he hasn't and all of those things are very important " RT13

Views on interacting with patients by mobile phone were mixed. Potential benefits were recognised:

"I do text, use mobile phone text. I've got some young asthmatic patients who don't ever come and see me unless they're exacerbated or that, you know, or their mothers phone me and say they're not very well... for the youngsters that's good. Even my asthmatic guy, who I hardly ever see, whenever I send him a text I always get a reply". RT15 However, feasibility was also questioned:

"....but I can't see how I would find the time to be texting people, to be honest, you know" RT17 Respondents also raised concerns about patients' willingness to accept new technologies which affected confidence in some of the applications, although some evidence indicated high patient acceptability:

"...but he found it (video link with clinical specialist nurse) absolutely excellent. He thought it was great to be in the comfort of his own home, speak to the nurse and get the answers that he was looking for, he loved

it, absolutely; his face was beaming" RT01

Skill set workability is concerned with allocation of tasks and resources, rewards and skill development, and with performance which is related to training and competence. For virtually all proposed developments there were significant concerns in this domain, often related to potential extra work by facilitating easier communication between patients and staff, or to adequacy of training. For example, in relation to email communication between clinician and patient, one clinician reported:

"Well, he was profoundly obsessional; would often email about his condition in quite a detailed way and he would go off to ..... for a few months and would send regular e-mail updates with questions that required careful consideration and reference to the notes, so it was actually pretty labour intensive without really much or any associated clinical benefit" RT12

The challenge of training was raised, describing potential difficulties:

" the concept of the untrainable, where no matter how often you show someone and explain it, they still don't get it right because they don't, you know, it's a foreign device to them; they don't like it" RT20

Contextual integration is concerned with execution, i.e. 
resource allocation, risk assessment and evaluation of effectiveness and with realisation i.e. shifting responsibilities between professional groupings and practical use of new resources. This aspect was not discussed for a number of the lower cost initiatives, but potential costs of initiatives that required substantial investment in equipment were repeatedly mentioned as a barrier to contextual integration. As well as costs, respondents raised the issue of the general strategic approach to telehealth initiatives, and whether the organisation was ready to implement on a wider scale:

".... and the lack of strategic overview is not helpful either about where they want telehealth to go, you know what is telehealth in Highland, do we actually know what we're talking about, are we talking about remote health monitoring and video cam technology ....... so we need to be able to define all the different types of telehealth solutions that we think we've already got or may want to start looking...." RT03 Finally, the importance of infrastructure was highlighted:

"... the one thing that will make it (telehealth) have more chance of success is if NHS Highland do set up infrastructure to support the delivery of that" RT03

\section{Discussion}

In this study, we examined the distribution of patients with common respiratory conditions across one health region in the UK and sought views from both primary and secondary care on the potential for telehealth applications. Current use of telehealth technologies in respiratory medicine is limited; ${ }^{2}$ evidence for their effective use is accruing, ${ }^{8-19}$ but there remain concerns about clinical and cost effectiveness. ${ }^{7,17}$ These issues will be affected by the setting in which telehealth is deployed.

Our patient distribution mapping indicated that a significant proportion of patients live in the most remote areas, including islands served by small general practices, and this distribution will impact on the design of telehealth services. Identifying patients from QOF data and treatment registers does not provide a true prevalence figure since it is dependent on case finding and accurate recording, but it does indicate the numbers of patients known to the service and can therefore provide a baseline for service profiling. It also does not take account of demand from tourists, nor provide an indication of disease severity - which will influence the types of service required. ${ }^{17}$ This would require further specific work prior to commissioning of local services. However, the high total numbers of patients living in more remote communities highlights the need to consider the available digital infrastructure - e.g. broadband and mobile phone connectivity - when planning potential service developments. Basic connectivity in the Highlands and Islands is below the UK average, ${ }^{26}$ and would currently be unable to support certain applications. This situation will be reflected in many rural and remote communities in the UK and worldwide.

Our sample of interviewees was relatively small $[n=20]$ but was purposively selected to provide a range of perspectives on telehealth developments. All interviewees, except the supplier, had direct responsibilities for primary or secondary care respiratory services across the region and all were enthusiastic about telehealth initiatives, citing potentially high levels of interactional workability and relational integration. This study identified some of the same barriers described in a previous survey published in 2004 involving remote practitioners. ${ }^{27}$ However, respondents to that survey had a more limited view of the value of telehealth, citing obtaining laboratory results by computer, transmission of electrocardiograms, and videoconferencing for education as potential applications, but raising many concerns about using telemedicine for clinical interaction with patients, including security and the negative impact on the quality of interaction.

The present study adopted a different methodology, and may be subject to some bias in that those interviewed might be likely to express more positive views of telehealth, although they were not selected with any prior knowledge of their attitudes to telehealth. It is possible that as computer technology and remote communications become more ubiquitous in other spheres such as banking and travel planning, health professionals are becoming more comfortable with the concept of telehealth. The recent successful introduction of a digital X-ray picture archiving system in our region, used by all hospital practitioners and some GPs, may have positively influenced practitioners' views of technology. Interestingly, our interviewees largely focussed on communication technologies, with some elements of management and information systems. This may reflect increasing emphasis on reduction of unnecessary travel, with its associated costs and environmental consequences.

The main perceived barriers to implementation in our study were in skill set workability - mainly training issues and contextual integration - mainly about costs. Current literature justifies legitimate concerns about cost issues. In a systematic review that identified 130 studies of home telehealth, only $17 \%$ included adequate economic data for assessment. ${ }^{28}$ As telehealth systems often have high capital costs associated with set-up, demonstrating cost benefit may take some years - although this can occur sooner where frequent hospital admissions are avoided. ${ }^{29}$ The lack of cost data could serve as a disincentive for healthcare providers to invest in new, as yet unproven, systems.

Interestingly, realisation - an element of contextual integration which refers to moving responsibilities between professional groups - was not a prominent concern, perhaps reflecting the extent to which this is already happening in rural communities as, for example, nurses and paramedics take on roles previously delivered by doctors. However, there is evidence 
that actual implementation of telecare services can face major problems of interactional workability where nurses lack confidence in the technological approach adopted. ${ }^{20}$

Developing recommendations for telehealth solutions also has to take account of wider considerations. For example, digital infrastructure is a key concern. There is a great potential risk that rural and remote areas, already disadvantaged by their physical geography, may remain disadvantaged by lack of connectivity one aspect of the "digital divide". ${ }^{30}$ Health care providers alone cannot solve this problem, which will require a joint approach across all sectors of society who stand to gain from improved connectivity.

Our study did not collect economic data, and this will require further study. However, current economic conditions are likely to present both a challenge and an opportunity to deliver services in a more efficient manner. Our study shows that there are substantial numbers of patients - both in urban and more remote locations - who could benefit, and a willingness by healthcare professionals to explore use of telehealth in these settings.

\section{Handling editor}

Onno van Schayck

\section{Statistical review}

Gopal Netuveli

\section{Acknowledgements}

We would like to acknowledge Dr Simon Evans, Dr Geoffrey Hulks, Dr Lorna Murray and Dr Stephen Thomas for their valuable contributions to the study; all the health professionals for agreeing to be interviewed; and lan Douglas for his help with mapping the Quality Outcomes Framework Registry data.

\section{Conflicts of interest}

The authors declare that they have no conflicts of interest in relation to this article.

\section{Contributorship}

David Godden conceived the study, analysed the quantitative data and co-wrote the manuscript. Gerry King designed the qualitative elements, performed interviews and analysis of transcripts, and co-wrote the manuscript.

\section{Funding}

We are grateful to the Sutherland Trust who funded this project.

\section{References}

1. The Burden of Lung Disease. 2nd Edition, The British Thoracic Society. London. 2006 accessed on 16/112010 at: http://www.britthoracic.org.uk/Portals/O/Library/ BTS\%20Publications/burdeon_of_lung_disease2007.pdf

2. Cooper C: Respiratory applications of telemedicine. Thorax 2009;64[3]:189-91. http://dx.doi.org/10.1136/thx.2008.104810

3. Mair FS, May C, Finch T, et al. Understanding the implementation and integration of e-health services. J Telemed Telecare 2007;13:S36-S37. http://dx.doi.org/10.1258/135763307781645112

4. Scottish Executive: Delivering for Health. Edinburgh; 2005. http://www.scotland.gov.uk/Publications/2005/11/02102635/26356

5. Scottish Executive: Building a Health Service Fit for the Future. Edinburgh; 2005, 39-43. http://www.scotland.gov.uk/Publications/2005/05/23141307/13104

6. Maclean S, Sheikh A. Does telehealthcare offer a patient-centred way forward for the community-based management of long-term respiratory disease? Prim Care Respir J 2009;18(3):125-6. http://dx.doi.org/10.3132/pcrj.2009.00006

7. Smith SM, Elkin SL, Partridge MR. Technology and its role in respiratory care. Prim Care Respir J 2009;18(3):159-64. http://dx.doi.org/10.4104/pcrj.2009.00038
8. Whitten P, Mickus M. Home telecare for COPD/CHF patients: outcomes and perceptions. J Telemed Telecare 2007;13(2):69-73. http://dx.doi.org/ $10.1258 / 135763307780096249$

9. Davey J. How collaborative care is becoming a reality in COPD management. Prim Health Care 2007;17(9):36-9.

10. Pfeifer M, Werner B, Magnussen $\mathrm{H}$. Telecare of patients with chronic obstructive airway diseases. Med Klin 2004;99(2):106-10. http://dx.doi.org/10.1007/s00063004-1019-4

11. Duvvuri VRSK, Jianhong W. Information and communication technology developments in asthma management: A systematic review. Indian J Med Sci 2007;61(4):221-41. http://dx.doi.org/10.4103/0019-5359.31159

12. Pinnock H, Shah R. Asthma. BMJ 2007;334:847-50. http://dx.doi.org/ 10.1136/bmj.39140.634896.BE.

13. Chan DS, Callahan CW, Hatch Pigott VB, et al. Internet-based home monitoring and education of children with asthma is comparable to ideal office-based care: results of a 1-year asthma in-home monitoring trial. Paediatrics 2007;119(3):56978. http://dx.doi.org/10.1542/peds.2006-1884

14. Maiolo C, Mohamed El, Fiorani CM, De Lorenzo A. Home telemonitoring for patients with severe respiratory illness: the Italian experience. J Telemed \& Telecare 2003;9(2):67-71. http://dx.doi.org/10.1258/135763303321327902

15. Kearney N, McCann L, Norrie J, et al. Evaluation of a mobile phone-based, advanced symptom management system (ASyMS) in the management of chemotherapy-related toxicity. Supportive Care in Cancer 2009;17(4):437-44. http://dx.doi.org/10.1007/s00520-008-0515-0

16. Pare $G$, Jaana $M$, Sicotte $C$. Systematic review of home telemonitoring for chronic diseases: the evidence base. Journal of the American Medical Informatics Association 2007;14(3):269-77. http://dx.doi.org/10.1197/jamia.M2270

17. McLean S, Chandler D, Nurmatov U, et al. Telehealthcare for asthma. Cochrane Database of Systematic Reviews 2010;10:CD0007717.

18. Agha Z, Schapira RM, Maker AH. Cost effectiveness of telemedicine for the delivery of outpatient pulmonary care to a rural population. Telemedicine Journal \& E-Health 2002;8[3]:281-91. http://dx.doi.org/10.1089/15305620260353171

19. Harno KS. Telemedicine in managing demand for secondary-care services. J Telemed and Telecare 1999;5[3]:189-92. http://dx.doi.org/10.1258/1357633991933611

20. Mair FS, Hiscock J, Beaton SC. Understanding factors that inhibit or promote the utilization of telecare in chronic lung disease. Chronic Illness 2008;4(2):110-17. http://dx.doi.org/10.1177/1742395308092482

21. NHS Scotland: Quality and Outcomes Framework. http://www.isdscotland.org/isd/3305.html

22. Swan GM, Selvaraj S, Godden DJ. Clinical peripherality: development of a peripherality index for rural health services. BMC Health Services Research 2008; 8:23. http://dx.doi.org/10.1186/1472-6963-8-23

23. Ritchie J, Spencer L. Qualitative data analysis for applied policy research. In: Bryman A, Burgess R, Eds. Analysing qualitative data. London, Routledge 1993, 173-194.

24. May C, Finch T, Mair $F$, et al. Understanding the implementation of complex interventions in health care: the normalization process model. BMC Health Services Research 2007;7:148. http://dx.doi.org/10.1186/1472-6963-7-148.

25. May, CR, Mair F, Finch T, et al. Development of a theory of implementation and integration: Normalization Process Theory. Implementation Science 2009;4:29. http://dx.doi.org/10.1186/1748-5908-4-29

26. Analysis Mason. Telecoms connectivity in the Highlands and Islands; 2009. Accessed 19/02/2010 at http://www.hie.co.uk/2009/Connectivity\%20 Review\%20-\%20Exec\%20Summary\%20Sept\%202009.pdf

27. Richards $H$, King G, Reid M, et al. Remote working: survey of attitudes to eHealth of doctors and nurses in rural general practices in the United Kingdom. Fam Pract 2004;21(6):1-6. http://dx.doi.org/10.1093/fampra/cmh71

28. Bensink M, Hailey $D$, Wootton R. A systematic review of successes and failures in home telehealth: preliminary results. J Telemed Telecare 2006;12(suppl_3):8-16. http://dx.doi.org/10.1258/135763306779380174

29. Seto E. Cost comparison between telemonitoring and usual care of heart failure: a systematic review. Telemedicine and e-Health 2008;14(7):679-83. http://dx.doi.org/10.1089/tmj.2007.0114

30. Commission for Rural Communities. Mind the Gap: Digital England - a Rural Perspective. London; 2009. http://www.ruralcommunities.gov.uk/files/ CRC104\%20Digital\%20Inclusion\%20Report.pdf 